\title{
Editorial
}

\section{Management of unstable angina: what role intervention, ask the RITA-3 trialists?}

Unstable angina usually results from the rupture of an atheromatous plaque within the coronary circulation, which provides a stimulus for platelet deposition and thrombosis. ${ }^{1}$ If the thrombus is subocclusive it produces intense regional ischaemia, expressed clinically as unstable angina, and there is an ill defined risk of progression to thrombotic coronary occlusion and myocardial infarction. Chest pain is inevitable and treatment with opiates should not be delayed. Nitrates and $\beta$ blockers are usually sufficient to correct myocardial ischaemia but dihydropyridine calcium antagonists (nifedipine, amlodipine) should be avoided, particularly in patients who are not taking $\beta$ blockers. $^{2}$

Because unstable angina is a thrombotic syndrome, treatment with antithrombotic drugs can reduce the risk of myocardial infarction and death. Thrombolytic therapy is unhelpful ${ }^{3}$ but three randomised trials have confirmed the benefits of aspirin for improving early prognosis. ${ }^{4-6}$ In one trial, unfractionated heparin was shown to have a similar beneficial effect, although there was no clear advantage over aspirin alone. ${ }^{6}$ Three further studies have confirmed that the combination of unfractionated heparin and aspirin confers little additional protection to that provided by aspirin alone..$^{7-9}$ Nevertheless, meta-analysis has suggested that unfractionated heparin may make a small independent contribution to risk reduction in unstable angina (risk ratio $0.67,95 \%$ confidence interval 0.44 to 1.02 ), and perhaps for this reason it remains widely used in combination with aspirin. ${ }^{10}$

A range of newer antithrombotic drugs has recently become available. Low molecular weight heparin, which has a more predictable anticoagulant effect than unfractionated heparin and can be given by subcutaneous injection without the need for anticoagulant monitoring, has been the subject of three large randomised trials in unstable angina. ${ }^{11-13}$ In two of them dalteparin and aspirin showed no clear benefit over aspirin alone ${ }^{11}$ or aspirin in combination with unfractionated heparin. ${ }^{12}$ In the third trial, enoxaparin in combination with aspirin resulted in a slightly lower combined incidence of death, myocardial infarction, and recurrent angina than occurred with unfractionated heparin and aspirin. ${ }^{13}$ It can be deduced, therefore, that low molecular weight heparin plus aspirin is at least as effective as unfractionated heparin plus aspirin in unstable angina, although there is no compelling evidence of added clinical benefit. It is likely that these comparative studies have overestimated the clinical value of unfractionated heparin, as proper anticoagulant monitoring (regular measurement of partial thromboplastin time with adjustment of infusion rate), which has been written into the study protocols, is rarely adhered to in clinical practice. Moreover, there is now evidence that the use of low molecular weight heparin may be associated with lower administration costs and a significant reduction in resource utilisation, particularly diagnostic cardiac catheterisation and angioplasty. ${ }^{14}{ }^{15}$ For these reasons, the argument for low molecular weight heparin in unstable angina is gaining momentum and it has already replaced unfractionated heparin in many coronary care units.
Potentially more interesting than low molecular weight heparin are drugs that inhibit platelet aggregation by antagonising the glycoprotein IIb/IIIa receptor on the platelet surface membrane. Only abciximab by intravenous infusion is currently available (ReoPro; Eli Lilly) and its value for protecting against major ischaemic events in high risk coronary angioplasty-a category that includes patients with unstable angina-is well established. ${ }^{16}{ }^{17}$ However, abciximab cannot be recommended for the routine management of unstable angina, although there is optimism that orally active IIb/IIIa receptor antagonists soon to become available may provide a useful addition to existing treatments. The recently licensed clopidogrel, which also interferes with platelet aggregation by inhibiting the binding of ADP to its platelet receptor, is being evaluated in unstable angina. Ticlopidine, its more toxic predecessor, has already been shown to be effective, but adverse side effects, particularly the risk of neutropenia, have limited its clinical application ${ }^{18}$ Clopidogrel has a safety profile equivalent to that of aspirin but appears to be more effective in protecting against ischaemic events in patients with atherosclerotic vascular disease. ${ }^{19}$ There is optimism therefore that it will find a useful role in the management of unstable angina.

Although the medical management of unstable angina is directed towards relieving pain and protecting against myocardial infarction and death, these aims cannot always be achieved. At least $13 \%$ of patients remain unstable after hospital admission with ongoing ischaemic chest pain. ${ }^{20}$ Moreover, in a review of 10 representative series with a total of nearly 2000 patients, the pooled one year mortality rate was estimated as $10 \%$ with a combined infarct and mortality rate of $21 \% .{ }^{21}$ Whether invasive management strategies involving coronary angiography and revascularisation provide the key to reducing event rates in unstable angina is an important question that remains unanswered. In many units, invasive management is reserved almost exclusively for patients with uncontrolled chest pain who cannot be discharged from hospital. Although these patients do appear to be at heightened risk, the policy is largely pragmatic and there is no clear evidence that it protects against future events. The ECG remains the best predictor of high risk. In a recent study, $14 \%$ of patients with ST depression on admission died or had a myocardial infarction within the 30 day follow up period. ${ }^{22}$ Risk stratification based on biochemical markers of myocardial injury has been the subject of recent investigation. Thus, increased serum concentrations of troponin $\mathrm{T}$ and $\mathrm{I}$ (structural proteins found only in cardiac myocytes) occur in about a third of patients with unstable angina and identify a group in whom morbidity and mortality are increased. ${ }^{23}{ }^{24}$ At present, there are no data regarding the value of invasive management in this high risk group.

In the absence of data to support a selective policy of invasive management in high risk patients, it is important to consider whether reductions in event rates might be achieved by a non-selective policy of invasive management applied to all patients with unstable angina. Generally, randomised trials in patients with acute coronary syndromes have been 
unable to demonstrate any clear advantage for invasive compared with conservative management strategies. ${ }^{35-27}$ However, only one of these trials recruited patients with unstable angina, and its clinical relevance is limited because a high crossover rate ensured that the proportion of patients undergoing revascularisation in the conservative group was almost as high as in the invasive group (49\% v 61\%), compromising the trial's ability to discriminate between the two strategies. ${ }^{3}$ The more recent report of non-randomised registry data in "organisation to assess strategies for ischemic syndromes" (OASIS) found that higher rates of invasive investigation and revascularisation were associated with a better symptomatic outcome but an increased rate of stroke, and did not appear to influence prognosis in terms of myocardial infarction and death. ${ }^{28}$

Continuing uncertainty about the role of cardiac catheterisation and revascularisation in the management of unstable angina largely accounts for the variations in clinical practice that exist between countries and individual physicians, some of whom recommend an invasive strategy for every case while others restrict it to the minority of patients with uncontrolled chest pain who cannot be discharged from hospital. ${ }^{28}$ There is no doubt that the use of invasive management in patients with unstable angina is increasing and using an increasing proportion of health care resources. It is therefore of considerable clinical and social importance to establish whether the invasive management of these patients influences clinical outcome. ${ }^{29}$ To answer this question the "randomised intervention treatment of angina" (RITA) trialists have embarked on a large multicentre study in the UK in which conservative and interventional treatment strategies are being compared in patients with unstable angina. In two previous landmark studies, the RITA trialists compared angioplasty with surgical ${ }^{30}$ and medical ${ }^{31}$ management of coronary artery disease. This, their third study, is no less important and deserves the support of all physicians involved in managing patients with unstable angina.

$S$ KENNON A TIMMIS

Department of Cardiology Royal Hospitals NHS Trust, London Chest Hospital, Bonner Road, London E2 97X, UK email:adam@timmis-lch.demon.co.uk

1 Davies MJ, Thomas AC. Plaque fissuring: the cause of acute myocardial infarction, sudden ischaemic death, and crescendo angina. Br Heart $\mathcal{f}$ 1983;50:127-34.

2 The Holland Interuniversity Nifedipine/Metoprolol Trial (HINT) Research Group. Early treatment of unstable angina in the coronary care unit: a randomised, double blind, placebo controlled comparison of recurrent ischaemia in patients treated with nifedipine or metoprolol or both. Br Heart $\mathcal{F}$ 1986;56:400-13.

3 The TIMI IIIB Investigators. Effects of tissue plasminogen activator and a comparison of early invasive and conservative strategies in unstable angina and non-Q-wave myocardial infarction. Results of the TIMI IIIB trial. Circulation 1994;89:1545-56.

4 Lewis HD, Davis JW, Archibald DG, et al. Protective effects of aspirin against acute myocardial infarction and death in men with unstable angina: results of a Veterans Administration cooperative study. N Engl F Med 1983; 309:396-403.

5 Cairns JA, Gent M, Singer J, et al. Aspirin, sulfinpyrazone, or both in unstable angina: results of a Canadian multicenter trial. $N$ Engl f Med 1985;313: 1369-75

6 Theroux P, Ouimet $\mathrm{H}$, McCans J, et al. Aspirin, heparin or both to treat unstable angina. N Engl f Med 1988;319:1105-11.

7 The RISC Group. Risk of myocardial infarction and death during treatment with low dose aspirin and intravenous heparin in men with unstable coronary artery disease. Lancet 1990;336:827-30.
8 Cohen M, Adams PC, Parry G, et al. and the Antithrombotic Therapy in Acute Coronary Syndromes (ATACS) Research Group. Combination antithrombotic therapy in unstable rest angina and non-Q-wave infarction in nonprior aspirin users. Circulation 1994;89:81-8.

9 Holdright D, Patel D, Cunningham D, et al. Comparison of the effect of heparin and aspirin versus aspirin alone on transient myocardial ischemia and in-hospital prognosis in patients with unstable angina. $7 \mathrm{Am}$ Coll Cardiol 1994;24:39-45.

10 Oler A, Whooley MA, Oler J, et al. Adding heparin to aspirin reduces the incidence of myocardial infarction and death in patients with unstable angina. $¥ A M A$ 1996;276:811-15.

11 Fragmin During Instability in Coronary Artery Disease (FRISC) Study Group. Low-molecular-weight heparin during instability in coronary artery disease. Lancet 1996;347:561-8.

12 Klein W, Buchwald A, Hillis SE, et al. Comparison of low-molecular-weight heparin with unfractionated heparin acutely and with placebo for 6 weeks in the management of unstable coronary artery disease: Fragmin in unstable coronary artery disease study (FRIC). Circulation 1997;96:61-8

13 Cohen M, Demers C, Gurfinkel EP, et al, for the Efficacy and Safety of Subcutaneous Enoxaparin in Non-Q-Wave Coronary Events (ESSENCE) Study Group. A comparison of low-molecular-weight heparin with unfractionated heparin for unstable coronary artery disease. N Engl f Med 1997; 337:447-52.

14 Mark D. When innovative therapies make economic sense: economic analysis of enoxaparin versus unfractionated heparin in the ESSENCE trial-an overview. Efficacy and safety of subcutaneous in non-Q wave coronary events. Can f Cardiol 1998;14(suppl E):24E-7E.

15 Fox KAA, Bosanquet N. Assessing the UK cost implications of the use of low molecular weight heparin in unstable coronary artery disease. $\mathrm{Br} \mathcal{F}$ Cardiol 1998;5:92-105.

16 The CAPTURE Investigators. Randomised placebo-controlled trial of abciximab before and during coronary intervention in refractory unstable angina: the CAPTURE study. Lancet 1997;349:1429-35.

17 The EPIC Investigators. Use of monoclonal antibody directed against the platelet glycoprotein IIb/IIIa receptor in high-risk coronary angioplasty. $N$ Engl F Med 1994;330:956-61.

18 Balsano F, Rizzon P, Violi F, et al. Antiplatelet treatment with ticlopidine in unstable angina. Circulation 1990;82:17-26.

19 A randomised, blinded, trial of clopidogrel versus aspirin in patients at risk of ischaemic events (CAPRIE). CAPRIE Steering Committee. Lancet 1996;348:1329-39.

20 Adams PC, Skinner JS, Cohen M, et al, and The Antithrombotic Therapy in Acute Coronary Syndromes Research Group. Acute coronary syndromes in the United States and United Kingdom: a comparison of approaches. Clin Cardiol 1998;21:348-52.

21 Betriu A, Heras $M$, Cohen $M$, et al. Unstable angina: outcome according to clinical presentation. 7 Am Coll Cardiol 1992;19:1659-63.

22 Holmvang L, Luscher MS, Clemmensen P, et al, and the TRIM Study Group. Very early risk stratification using combined ECG and biochemical assessment in patients with unstable coronary artery disease. (A thrombin inhibition in myocardial ischaemia (TRIM) substudy). Circulation 1998;98: 2004-9

23 Lindahl B, Venge P, Wallentin L. Troponin T identifies patients with unstable coronary artery disease who benefit from long-term antithrombotic protection. Fragmin in Unstable Coronary Artery Disease (FRISC) Study Group. 7 Am Coll Cardiol 1997;29:43-8.

24 Mockel M, Stork T, Heller G, et al. Troponin T in patients with low grade or atypical angina. Identification of a high risk group for short- and long-term cardiovascular events. Eur Heart $\mathcal{F}$ 1998;19:1802-7.

25 The TIMI Study Group. Comparison of invasive and conservative strategies after treatment with intravenous tissue plasminogen activator in acute myocardial infarction: results of the thrombolysis in myocardial infarction (TIMI) phase II trial. N Engl f Med 1989;320:618-27.

26 SWIFT (Should We Intervene Following Thrombolysis?) Trial Study Group. SWIFT trial of delayed elective intervention $v$ conservative treatment after thrombolysis with anistrplase in acute myocardial infarction. BMF 1991;302:555-60.

27 Boden WE, Rourke RA, Crawford $\mathrm{MH}$, et al. Outcomes in patients with acute non-Q-wave myocardial infarction randomly assigned to an invasive as compared with a conservative management strategy. N Engl f Med 1998; 338:1785-92.

28 Yusuf S, Pogue J, Hunt D, et al, for the OASIS (Organisation to Assess Strategies for Ischemic Syndromes) Registry Investigators. Variations between countries in invasive cardiac procedures and outcomes in patients with suspected unstable angina or myocardial infarction without initial ST elevation. Lancet 1998;352:507-14.

29 Topol E. What role for catheter laboratories in unstable angina. Lancet 1998;352:500-1.

30 RITA Trial Participants. Coronary angioplasty versus coronary artery bypass surgery: the randomised intervention treatment of angina (RITA) trial. Lancet 1993;341:573-80.

31 RITA-2 Trial Participants. Coronary angioplasty versus medical therapy for angina: the second randomised intervention treatment of angina (RITA-2) trial. Lancet 1997;350:461-8. 